\title{
Posterior calibration and exploratory analysis for natural language processing models
}

\author{
Khanh Nguyen \\ Department of Computer Science \\ University of Maryland, College Park \\ College Park, MD 20742 \\ kxnguyen@cs. umd.edu
}

\author{
Brendan O'Connor \\ College of Information and Computer Sciences \\ University of Massachusetts, Amherst \\ Amherst, MA, 01003 \\ brenoconecs.umass.edu
}

\begin{abstract}
Many models in natural language processing define probabilistic distributions over linguistic structures. We argue that (1) the quality of a model's posterior distribution can and should be directly evaluated, as to whether probabilities correspond to empirical frequencies; and (2) NLP uncertainty can be projected not only to pipeline components, but also to exploratory data analysis, telling a user when to trust and not trust the NLP analysis. We present a method to analyze calibration, and apply it to compare the miscalibration of several commonly used models. We also contribute a coreference sampling algorithm that can create confidence intervals for a political event extraction task. ${ }^{1}$
\end{abstract}

\section{Introduction}

Natural language processing systems are imperfect. Decades of research have yielded analyzers that mis-identify named entities, mis-attach syntactic relations, and mis-recognize noun phrase coreference anywhere from $10-40 \%$ of the time. But these systems are accurate enough so that their outputs can be used as soft, if noisy, indicators of language meaning for use in downstream analysis, such as systems that perform question answering, machine translation, event extraction, and narrative analysis (McCord et al., 2012; Gimpel and Smith, 2008; Miwa et al., 2010; Bamman et al., 2013).

To understand the performance of an analyzer, researchers and practitioners typically measure the accuracy of individual labels or edges among a single predicted output structure $\mathbf{y}$, such as a most-probable tagging or entity clustering $\arg \max _{\mathbf{y}} P(\mathbf{y} \mid x)$ (conditional on text data $x$ ).

\footnotetext{
${ }^{1}$ See the extended version of this paper for software, appendix, and acknowledgments sections:

http://brenocon.com/nlpcalib/

http://arxiv.org/abs/1508.05154
}

But a probabilistic model gives a probability distribution over many other output structures that have smaller predicted probabilities; a line of work has sought to control cascading pipeline errors by passing on multiple structures from earlier stages of analysis, by propagating prediction uncertainty through multiple samples (Finkel et al., 2006), $K$-best lists (Venugopal et al., 2008; Toutanova et al., 2008), or explicitly diverse lists (Gimpel et al., 2013); often the goal is to marginalize over structures to calculate and minimize an expected loss function, as in minimum Bayes risk decoding (Goodman, 1996; Kumar and Byrne, 2004), or to perform joint inference between early and later stages of NLP analysis (e.g. Singh et al., 2013; Durrett and Klein, 2014).

These approaches should work better when the posterior probabilities of the predicted linguistic structures reflect actual probabilities of the structures or aspects of the structures. For example, say a model is overconfident: it places too much probability mass in the top prediction, and not enough in the rest. Then there will be little benefit to using the lower probability structures, since in the training or inference objectives they will be incorrectly outweighed by the top prediction (or in a sampling approach, they will be systematically undersampled and thus have too-low frequencies). If we only evaluate models based on their top predictions or on downstream tasks, it is difficult to diagnose this issue.

Instead, we propose to directly evaluate the calibration of a model's posterior prediction distribution. A perfectly calibrated model knows how often it's right or wrong; when it predicts an event with $80 \%$ confidence, the event empirically turns out to be true $80 \%$ of the time. While perfect accuracy for NLP models remains an unsolved challenge, perfect calibration is a more achievable goal, since a model that has imperfect accuracy could, in principle, be perfectly calibrated. In this paper, we develop a method to empirically analyze calibration that is appropriate for NLP models (§3) 
and use it to analyze common generative and discriminative models for tagging and classification (§4).

Furthermore, if a model's probabilities are meaningful, that would justify using its probability distributions for any downstream purpose, including exploratory analysis on unlabeled data. In $\S 6$ we introduce a representative corpus exploration problem, identifying temporal event trends in international politics, with a method that is dependent on coreference resolution. We develop a coreference sampling algorithm (§5.2) which projects uncertainty into the event extraction, inducing a posterior distribution over event frequencies. Sometimes the event trends have very high posterior variance (large confidence intervals), ${ }^{2}$ reflecting when the NLP system genuinely does not know the correct semantic extraction. This highlights an important use of a calibrated model: being able to tell a user when the model's predictions are likely to be incorrect, or at least, not giving a user a false sense of certainty from an erroneous NLP analysis.

\section{Definition of calibration}

Consider a binary probabilistic prediction problem, which consists of binary labels and probabilistic predictions for them. Each instance has a ground-truth label $y \in\{0,1\}$, which is used for evaluation. The prediction problem is to generate a predicted probability or prediction strength $q \in[0,1]$. Typically, we use some form of a probabilistic model to accomplish this task, where $q$ represents the model's posterior probability ${ }^{3}$ of the instance having a positive label $(y=1)$.

Let $S=\left\{\left(q_{1}, y_{1}\right),\left(q_{2}, y_{2}\right), \cdots\left(q_{N}, y_{N}\right)\right\}$ be the set of prediction-label pairs produced by the model. Many metrics assess the overall quality of how well the predicted probabilities match the data, such as the familiar cross entropy (negative average log-likelihood),

$$
L_{\ell}(\vec{y}, \vec{q})=\frac{1}{N} \sum_{i} y_{i} \log \frac{1}{q_{i}}+\left(1-y_{i}\right) \log \frac{1}{1-q_{i}}
$$

or mean squared error, also known as the Brier score when $y$ is binary (Brier, 1950),

$$
L_{2}(\vec{y}, \vec{q})=\frac{1}{N} \sum_{i}\left(y_{i}-q_{i}\right)^{2}
$$

\footnotetext{
${ }^{2}$ We use the terms confidence interval and credible interval interchangeably in this work; the latter term is debatably more correct, though less widely familiar.

${ }^{3}$ Whether $q$ comes from a Bayesian posterior or not is irrelevant to the analysis in this section. All that matters is that predictions are numbers $q \in[0,1]$.
}

Both tend to attain better (lower) values when $q$ is near 1 when $y=1$, and near 0 when $y=0$; and they achieve a perfect value of 0 when all $q_{i}=y_{i}{ }^{4}$

Let $\mathbb{P}(y, q)$ be the joint empirical distribution over labels and predictions. Under this notation, $L_{2}=\mathbb{E}_{q, y}[y-q]^{2}$. Consider the factorization

$$
\mathbb{P}(y, q)=\mathbb{P}(y \mid q) \mathbb{P}(q)
$$

where $\mathbb{P}(y \mid q)$ denotes the label empirical frequency, conditional on a prediction strength (Murphy and Winkler, 1987). ${ }^{5}$ Applying this factorization to the Brier score leads to the calibrationrefinement decomposition (DeGroot and Fienberg, 1983), in terms of expectations with respect to the prediction strength distribution $\mathbb{P}(q)$ :

$$
L_{2}=\underbrace{\mathbb{E}_{q}\left[q-p_{q}\right]^{2}}_{\text {Calibration MSE }}+\underbrace{\mathbb{E}_{q}\left[p_{q}\left(1-p_{q}\right)\right]}_{\text {Refinement }}
$$

where we denote $p_{q} \equiv \mathbb{P}(y=1 \mid q)$ for brevity.

Here, calibration measures to what extent a model's probabilistic predictions match their corresponding empirical frequencies. Perfect calibration is achieved when $\mathbb{P}(y=1 \mid q)=q$ for all $q$; intuitively, if you aggregate all instances where a model predicted $q$, they should have $y=1$ at $q$ percent of the time. We define the magnitude of miscalibration using root mean squared error:

Definition 1 (RMS calibration error).

$$
\text { CalibErr }=\sqrt{\mathbb{E}_{q}[q-\mathbb{P}(y=1 \mid q)]^{2}}
$$

The second term of Eq 1 refers to refinement, which reflects to what extent the model is able to separate different labels (in terms of the conditional Gini entropy $p_{q}\left(1-p_{q}\right)$ ). If the prediction strengths tend to cluster around 0 or 1 , the refinement score tends to be lower. The calibrationrefinement breakdown offers a useful perspective on the accuracy of a model posterior. This paper focuses on calibration.

There are several other ways to break down squared error, log-likelihood, and other probabilistic scoring rules. ${ }^{6}$ We use the Brier-based calibration error in this work, since unlike cross-entropy

\footnotetext{
${ }^{4}$ These two loss functions are instances of proper scoring rules (Gneiting and Raftery, 2007; Bröcker, 2009).

${ }^{5}$ We alternatively refer to this as label frequency or empirical frequency. The $\mathbb{P}$ probabilities can be thought of as frequencies from the hypothetical population the data and predictions are drawn from. $\mathbb{P}$ probabilities are, definitionally speaking, completely separate from a probabilistic model that might be used to generate $q$ predictions.

${ }^{6}$ They all include a notion of calibration corresponding to a Bregman divergence (Bröcker, 2009); for example, crossentropy can be broken down such that KL divergence is the measure of miscalibration.
} 
$\overline{\text { Algorithm } 1 \text { Estimate calibration error using }}$ adaptive binning.

Input: A set of $N$ prediction-label pairs

$\left\{\left(q_{1}, y_{1}\right),\left(q_{2}, y_{2}\right), \cdots,\left(q_{N}, y_{N}\right)\right\}$.

Output: Calibration error.

Parameter: Target bin size $\beta$.

Step 1: Sort pairs by prediction values $q_{k}$ in ascending order.

Step 2: For each, assign bin label $b_{k}=\left\lfloor\frac{k-1}{\beta}\right\rfloor+1$.

Step 3: Define each bin $B_{i}$ as the set of indices of pairs that have the same bin label. If the last bin has size less than $\beta$, merge it with the second-to-last bin (if one exists). Let $\left\{B_{1}, B_{2}, \cdots, B_{T}\right\}$ be the set of bins.

Step 4: Calculate empirical and predicted probabilities per bin:

$$
\hat{p}_{i}=\frac{1}{\left|B_{i}\right|} \sum_{k \in B_{i}} y_{k} \quad \text { and } \quad \hat{q}_{i}=\frac{1}{\left|B_{i}\right|} \sum_{k \in B_{i}} q_{k}
$$

Step 5: Calculate the calibration error as the root mean squared error per bin, weighted by bin size in case they are not uniformly sized:

$$
\text { CalibErr }=\sqrt{\frac{1}{N} \sum_{i=1}^{T}\left|B_{i}\right|\left(\hat{q}_{i}-\hat{p}_{i}\right)^{2}}
$$

it does not tend toward infinity when near probability 0 ; we hypothesize this could be an issue since both $p$ and $q$ are subject to estimation error.

\section{Empirical calibration analysis}

From a test set of labeled data, we can analyze model calibration both in terms of the calibration error, as well as visualizing the calibration curve of label frequency versus predicted strength. However, computing the label frequencies $\mathbb{P}(y=1 \mid q)$ requires an infinite amount of data. Thus approximation methods are required to perform calibration analysis.

\subsection{Adaptive binning procedure}

Previous studies that assess calibration in supervised machine learning models (Niculescu-Mizil and Caruana, 2005; Bennett, 2000) calculate label frequencies by dividing the prediction space into deciles or other evenly spaced bins-e.g. $q \in$ $[0,0.1), q \in[0.1,0.2)$, etc. - and then calculating the empirical label frequency in each bin. This procedure may be thought of as using a form of nonparametric regression (specifically, a regressogram; Tukey 1961) to estimate the function $f(q)=\mathbb{P}(y=1 \mid q)$ from observed data points. But models in natural language processing give very skewed distributions of confidence scores $q$ (many are near 0 or 1 ), so this procedure performs poorly, having much more variable estimates near
Algorithm 2 Estimate calibration error's confidence interval by sampling.

Input: $\quad \mathrm{A}$ set of $N$ prediction-label pairs $\left\{\left(q_{1}, y_{1}\right),\left(q_{2}, y_{2}\right), \cdots,\left(q_{N}, y_{N}\right)\right\}$.

Output: Calibration error with a $95 \%$ confidence interval.

Parameter: Number of samples, $S$.

Step 1: Calculate $\left\{\hat{p}_{1}, \hat{p}_{2}, \cdots, \hat{p}_{T}\right\}$ from step 4 of Algorithm 1.

Step 2: Draw $S$ samples. For each $s=1 . . S$,

- For each bin $i=1 . . T$, draw $\hat{p}_{i}^{(s)} \sim \mathcal{N}\left(\hat{p}_{i}, \hat{\sigma}_{i}^{2}\right)$, where $\hat{\sigma}_{i}^{2}=\hat{p}_{i}\left(1-\hat{p}_{i}\right) /\left|B_{i}\right|$. If necessary clip to $[0,1]$ : $\hat{p}_{i}^{(s)}:=\min \left(1, \max \left(0, \hat{p}_{i}^{(s)}\right)\right)$

- Calculate the sample's CalibErr from using the pairs $\left(\hat{q}_{i}, \hat{p}_{i}^{(s)}\right)$ as per Step 5 of Algorithm 1 .

Step 3: Calculate the $95 \%$ confidence interval for the calibration error as:

$$
\text { CalibErr }{ }_{\text {avg }} \pm 1.96 \hat{s}_{\text {error }}
$$

where CalibErr ${ }_{\text {avg }}$ and $\hat{s}_{\text {error }}$ are the mean and the standard deviation, respectively, of the CalibErrs calculated from the samples.

the middle of the $q$ distribution (Figure 1).

We propose adaptive binning as an alternative. Instead of dividing the interval $[0,1]$ into fixed-width bins, adaptive binning defines the bins such that there are an equal number of points in each, after which the same averaging procedure is used. This method naturally gives wider bins to area with fewer data points (areas that require more smoothing), and ensures that these areas have roughly similar standard errors as those near the boundaries, since for a bin with $\beta$ number of points and empirical frequency $p$, the standard error is estimated by $\sqrt{p(1-p) / \beta}$, which is bounded above by $0.5 / \sqrt{\beta}$. Algorithm 1 describes the procedure for estimating calibration error using adaptive binning, which can be applied to any probabilistic model that predicts posterior probabilities.

\subsection{Confidence interval estimation}

Especially when the test set is small, estimating calibration error may be subject to error, due to uncertainty in the label frequency estimates. Since how to estimate confidence bands for nonparametric regression is an unsolved problem (Wasserman, 2006), we resort to a simple method based on the binning. We construct a binomial normal approximation for the label frequency estimate in each bin, and simulate from it; every simulation across all bins is used to construct a calibration error; these simulated calibration errors are collected to construct a normal approximation for the calibra- 


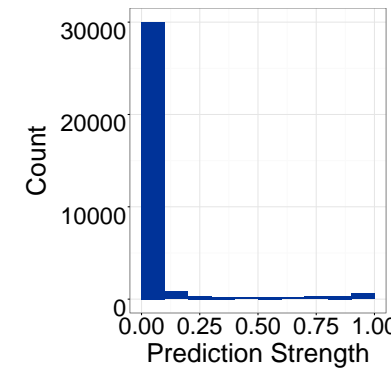

(a)

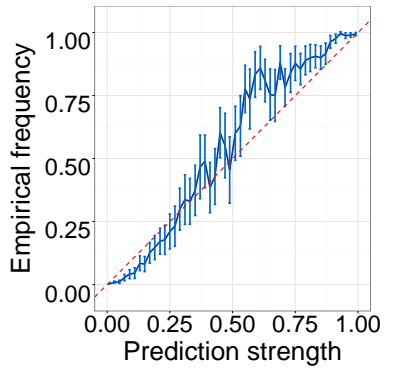

(b)

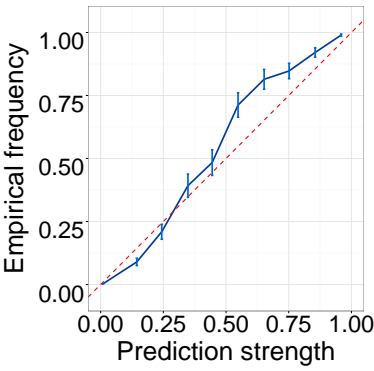

(c)

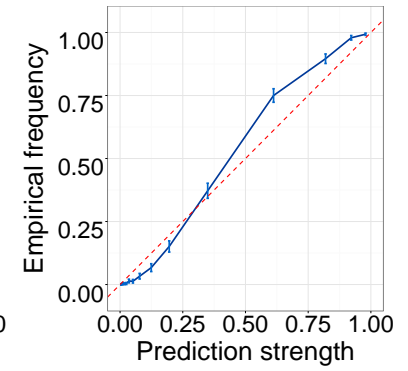

(d)

Figure 1: (a) A skewed distribution of predictions on whether a word has the NN tag (§4.2.2). Calibration curves produced by equally-spaced binning with bin width equal to 0.02 (b) and 0.1 (c) can have wide confidence intervals. Adaptive binning (with 1000 points in each bin) (d) gives small confidence intervals and also captures the prediction distribution. The confidence intervals are estimated as described in $\S 3.1$.

tion error estimate. Since we use bin sizes of at least $\beta \geq 200$ in our experiments, the central limit theorem justifies these approximations. We report all calibration errors along with their $95 \%$ confidence intervals calculated by Algorithm 2. ${ }^{7}$

\subsection{Visualizing calibration}

In order to better understand a model's calibration properties, we plot the pairs $\left(\hat{p}_{1}, \hat{q}_{1}\right),\left(\hat{p}_{2}, \hat{q}_{2}\right), \cdots,\left(\hat{p}_{T}, \hat{q}_{T}\right)$ obtained from the adaptive binning procedure to visualize the calibration curve of the model - this visualization is known as a calibration or reliability plot. It provides finer grained insight into the calibration behavior in different prediction ranges. A perfectly calibrated curve would coincide with the $y=x$ diagonal line. When the curve lies above the diagonal, the model is underconfident $\left(q<p_{q}\right)$; and when it is below the diagonal, the model is overconfident $\left(q>p_{q}\right)$.

An advantage of plotting a curve estimated from fixed-size bins, instead of fixed-width bins, is that the distribution of the points hints at the refinement aspect of the model's performance. If the points' positions tend to cluster in the bottom-left and topright corners, that implies the model is making more refined predictions.

\section{Calibration for classification and tagging models}

Using the method described in $\S 3$, we assess the quality of posterior predictions of several classification and tagging models. In all of our exper-

\footnotetext{
${ }^{7}$ A major unsolved issue is how to fairly select the bin size. If it is too large, the curve is oversmoothed and calibration looks better than it should be; if it is too small, calibration looks worse than it should be. Bandwidth selection and cross-validation techniques may better address this problem in future work. In the meantime, visualizations of calibration curves help inform the reader of the resolution of a particular analysis - if the bins are far apart, the data is sparse, and the specific details of the curve are not known in those regions.
}

iments, we set the target bin size in Algorithm 1 to be 5,000 and the number of samples in Algorithm 2 to be 10,000 .

\subsection{Naive Bayes and logistic regression}

\subsubsection{Introduction}

Previous work on Naive Bayes has found its probabilities to have calibration issues, in part due to its incorrect conditional independence assumptions (Niculescu-Mizil and Caruana, 2005; Bennett, 2000; Domingos and Pazzani, 1997). Since logistic regression has the same log-linear representational capacity ( $\mathrm{Ng}$ and Jordan, 2002) but does not suffer from the independence assumptions, we select it for comparison, hypothesizing it may have better calibration.

We analyze a binary classification task of Twitter sentiment analysis from emoticons. We collect a dataset consisting of tweets identified by the Twitter API as English, collected from 2014 to 2015, with the "emoticon trick" (Read, 2005; Lin and Kolcz, 2012) to label tweets that contain at least one occurrence of the smiley emoticon ":)" as "happy" $(y=1)$ and others as $y=0$. The smiley emoticons are deleted in positive examples. We sampled three sets of tweets (subsampled from the Decahose/Gardenhose stream of public tweets) with Jan-Apr 2014 for training, May-Dec 2014 for development, and Jan-Apr 2015 for testing. Each set contains $10^{5}$ tweets, split between an equal number of positive and negative instances. We use binary features based on unigrams extracted from the twokenize.py ${ }^{8}$ tokenization. We use the scikit-learn (Pedregosa et al., 2011) implementations of Bernoulli Naive Bayes and L2-regularized logistic regression. The models' hyperparameters (Naive Bayes' smoothing paramter and logistic regression's regularization strength) are chosen to

\footnotetext{
${ }^{8}$ https://github.com/myleott/ ark-twokenize-py
} 


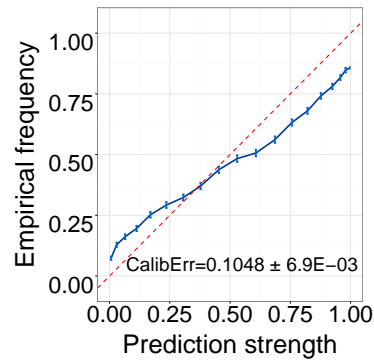

(a)

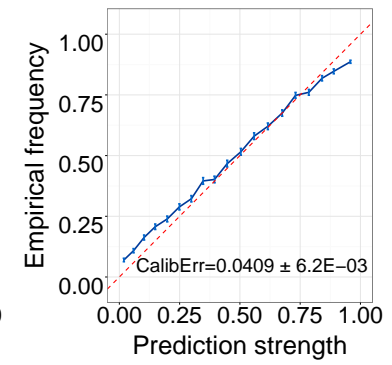

(b)
Figure 2: Calibration curve of (a) Naive Bayes and (b) logistic regression on predicting whether a tweet is a "happy" tweet.

maximize the F-1 score on the development set.

\subsubsection{Results}

Naive Bayes attains a slightly higher F-1 score (NB 73.8\% vs. LR 72.9\%), but logistic regression has much lower calibration error: less than half as much RMSE (NB 0.105 vs. LR 0.041; Figure 2 ). Both models have a tendency to be underconfident in the lower prediction range and overconfident in the higher range, but the tendency is more pronounced for Naive Bayes.

\subsection{Hidden Markov models and conditional random fields}

\subsubsection{Introduction}

Hidden Markov models (HMM) and linear chain conditional random fields (CRF) are another commonly used pair of analogous generative and discriminative models. They both define a posterior over tag sequences $P(\mathbf{y} \mid x)$, which we apply to part-of-speech tagging.

We can analyze these models in the binary calibration framework (§2-3) by looking at marginal distribution of binary-valued outcomes of parts of the predicted structures. Specifically, we examine calibration of predicted probabilities of individual tokens' tags (§4.2.2), and of pairs of consecutive tags (§4.2.3). These quantities are calculated with the forward-backward algorithm.

To prepare a POS tagging dataset, we extract Wall Street Journal articles from the English CoNLL-2011 coreference shared task dataset from Ontonotes (Pradhan et al., 2011), using the CoNLL-2011 splits for training, development and testing. This results in 11,772 sentences for training, 1,632 for development, and 1,382 for testing, over a set of 47 possible tags.

We train an HMM with Dirichlet MAP using one pseudocount for every transition and word emission. For the CRF, we use the $L_{2}$ regularized L-BFGS algorithm implemented in

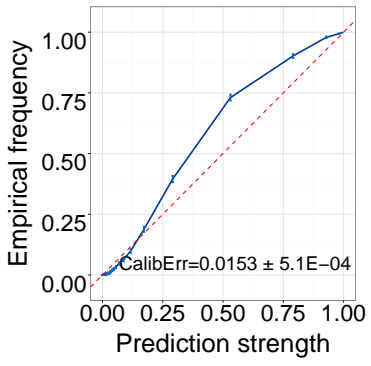

(a)

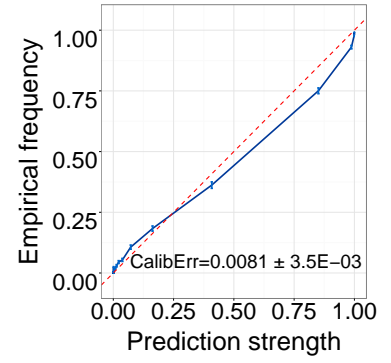

(b)
Figure 3: Calibration curves of (a) HMM, and (b) CRF, on predictions over all POS tags.

CRFsuite (Okazaki, 2007). We compare an HMM to a CRF that only uses basic transition (tag-tag) and emission (tag-word) features, so that it does not have an advantage due to more features. In order to compare models with similar task performance, we train the CRF with only 3000 sentences from the training set, which yields the same accuracy as the HMM (about $88.7 \%$ on the test set). In each case, the model's hyperparameters (the CRF's $L_{2}$ regularizer, the HMM's pseudocount) are selected by maximizing accuracy on the development set.

\subsubsection{Predicting single-word tags}

In this experiment, we measure miscalibration of the two models on predicting tags of single words. First, for each tag type, we produce a set of 33,306 prediction-label pairs (for every token); we then concatenate them across the tags for calibration analysis. Figure 3 shows that the two models exhibit distinct calibration patterns. The HMM tends to be very underconfident whereas the CRF is overconfident, and the CRF has a lower (better) overall calibration error.

We also examine the calibration errors of the individual POS tags (Figure 4(a)). We find that CRF is significantly better calibrated than HMM in most but not all categories (39 out of 47). For example, they are about equally calibrated on predicting the NN tag. The calibration gap between the two models also differs among the tags.

\subsubsection{Predicting two-consecutive-word tags}

There is no reason to restrict ourselves to model predictions of single words; these models define marginal distributions over larger textual units. Next we examine the calibration of posterior predictions of tag pairs on two consecutive words in the test set. The same analysis may be important for, say, phrase extraction or other chunking/parsing tasks. 


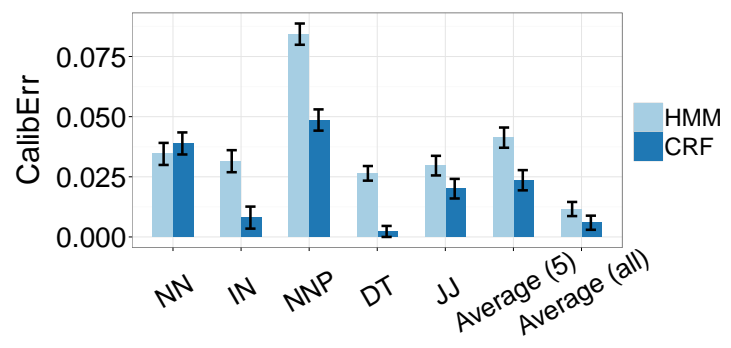

(a)

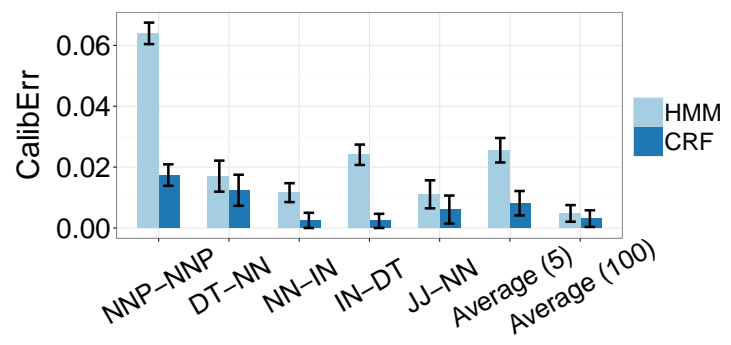

(b)

Figure 4: Calibration errors of HMM and CRF on predicting (a) single-word tags and (b) two-consecutive-word tags. Lower errors are better. The last two columns in each graph are the average calibration errors over the most common labels.

We report results for the top 5 and 100 most frequent tag pairs (Figure 4(b)). We observe a similar pattern as seen from the experiment on single tags: the CRF is generally better calibrated than the HMM, but the HMM does achieve better calibration errors in 29 out of 100 categories.

These tagging experiments illustrate that, depending on the application, different models can exhibit different levels of calibration.

\section{Coreference resolution}

We examine a third model, a probabilistic model for within-document noun phrase coreference, which has an efficient sampling-based inference procedure. In this section we introduce it and analyze its calibration, in preparation for the next section where we use it for exploratory data analysis.

\subsection{Antecedent selection model}

We use the Berkeley coreference resolution system (Durrett and Klein, 2013), which was originally presented as a CRF; we give it an equivalent a series of independent logistic regressions (see appendix for details). The primary component of this model is a locally-normalized log-linear distribution over clusterings of noun phrases, each cluster denoting an entity. The model takes a fixed input of $N$ mentions (noun phrases), indexed by $i$ in their positional order in the document. It posits that every mention $i$ has a latent antecedent selection decision, $a_{i} \in\{1, \ldots, i-1, \mathrm{NEW}\}$, denoting which previous mention it attaches to, or NEW if it is starting a new entity that has not yet been seen at a previous position in the text. Such a mentionmention attachment indicates coreference, while the final entity clustering includes more links implied through transitivity. The model's generative process is:

Definition 2 (Antencedent coreference model and sampling algorithm).

- For $i=1 . . N$, sample $a_{i} \sim \frac{1}{Z_{i}} \exp \left(\mathbf{w}^{\top} \mathbf{f}\left(i, a_{i}, x\right)\right)$

- Calculate the entity clusters as $\mathbf{e}:=C C(\mathbf{a})$, the connected components of the antecedent graph having edges $\left(i, a_{i}\right)$ for $i$ where $a_{i} \neq$ NEW.

Here $x$ denotes all information in the document that is conditioned on for log-linear features $f$. $\mathbf{e}=\left\{e_{1}, \ldots e_{M}\right\}$ denotes the entity clusters, where each element is a set of mentions. There are $M$ entity clusters corresponding to the number of connected components in $\mathbf{a}$. The model defines a joint distribution over antecedent decisions $P(\mathbf{a} \mid x)=$ $\prod_{i} P\left(a_{i} \mid x\right)$; it also defines a joint distribution over entity clusterings $P(\mathbf{e} \mid x)$, where the probability of an $\mathbf{e}$ is the sum of the probabilities of all a vectors that could give rise to it. In a manner similar to a distance-dependent Chinese restaurant process (Blei and Frazier, 2011), it is non-parametric in the sense that the number of clusters $M$ is not fixed in advance.

\subsection{Sampling-based inference}

For both calibration analysis and exploratory applications, we need to analyze the posterior distribution over entity clusterings. This distribution is a complex mathematical object; an attractive approach to analyze it is to draw samples from this distribution, then analyze the samples.

This antecedent-based model admits a very straightforward procedure to draw independent $\mathbf{e}$ samples, by stepping through Def. 2: independently sample each $a_{i}$ then calculate the connected components of the resulting antecedent graph. By construction, this procedure samples from the joint distribution of e (even though we never compute the probability of any single clustering e).

Unlike approximate sampling approaches, such as Markov chain Monte Carlo methods used in other coreference work to sample e (Haghighi and Klein, 2007), here there are no questions about burn-in or autocorrelation (Kass et al., 1998). Every sample is independent and very fast to 


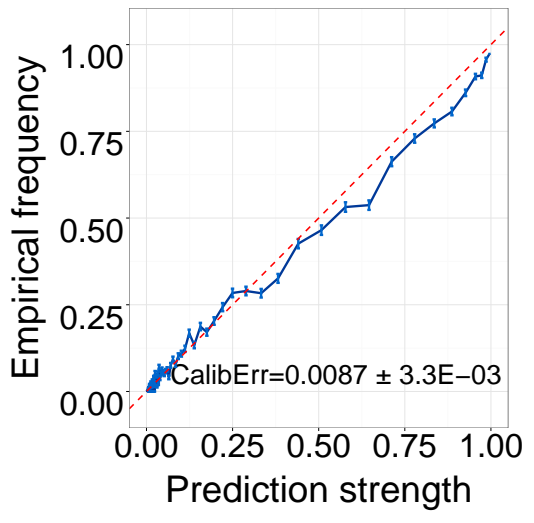

Figure 5: Coreference calibration curve for predicting whether two mentions belong to the same entity cluster.

compute-only slightly slower than calculating the MAP assignment (due to the exp and normalization for each $a_{i}$ ). We implement this algorithm by modifying the publicly available implementation from Durrett and Klein. ${ }^{9}$

\subsection{Calibration analysis}

We consider the following inference query: for a randomly chosen pair of mentions, are they coreferent? Even if the model's accuracy is comparatively low, it may be the case that it is correctly calibrated-if it thinks there should be great variability in entity clusterings, it may be uncertain whether a pair of mentions should belong together.

Let $\ell_{i j}$ be 1 if the mentions $i$ and $j$ are predicted to be coreferent, and 0 otherwise. Annotated data defines a gold-standard $\ell_{i j}^{(g)}$ value for every pair $i, j$. Any probability distribution over e defines a marginal Bernoulli distribution for every proposition $\ell_{i j}$, marginalizing out $\mathbf{e}$ :

$$
P\left(\ell_{i j}=1 \mid x\right)=\sum_{\mathbf{e}} 1\{(i, j) \in \mathbf{e}\} P(\mathbf{e} \mid x)
$$

where $(i, j) \in \mathbf{e}$ is true iff there is an entity in $\mathbf{e}$ that contains both $i$ and $j$.

In a traditional coreference evaluation of the best-prediction entity clustering, the model assigns 1 or 0 to every $\ell_{i j}$ and the pairwise precision and recall can be computed by comparing them to the corresponding $\ell_{i j}^{(g)}$. Here, we instead compare the $q_{i j} \equiv P\left(\ell_{i j}=1 \mid x, \mathbf{e}\right)$ prediction strengths against $\ell_{i j}^{(g)}$ empirical frequencies to assess pairwise calibration, with the same binary calibration analysis tools developed in $\S 3$ by aggregating pairs with similar $q_{i j}$ values. Each $q_{i j}$ is computed by averaging over 1,000 samples, simply taking the fraction of samples where the pair $(i, j)$ is coreferent.

\footnotetext{
${ }^{9}$ Berkeley Coreference Resolution System, version 1.1: http://nlp.cs.berkeley.edu/projects/ coref.shtml
}

We perform this analysis on the development section of the English CoNLL-2011 data (404 documents). Using the sampling inference method discussed in $\S 5.2$, we compute 4.3 millions prediction-label pairs and measure their calibration error. Our result shows that the model produces very well-calibrated predictions with less than $1 \%$ CalibErr (Figure 5), though slightly overconfident on middle to high-valued predictions. The calibration error indicates that it is the most calibrated model we examine within this paper. This result suggests we might be able to trust its level of uncertainty.

\section{Uncertainty in Entity-based Exploratory Analysis}

\subsection{Entity-syntactic event aggregation}

We demonstrate one important use of calibration analysis: to ensure the usefulness of propagating uncertainty from coreference resolution into a system for exploring unannotated text. Accuracy cannot be calculated since there are no labels; but if the system is calibrated, we postulate that uncertainty information can help users understand the underlying reliability of aggregated extractions and isolate predictions that are more likely to contain errors.

We illustrate with an event analysis application to count the number of "country attack events": for a particular country of the world, how many news articles describe an entity affiliated with that country as the agent of an attack, and how does this number change over time? This is a simplified version of a problem where such systems have been built and used for political science analysis (Schrodt et al., 1994; Schrodt, 2012; Leetaru and Schrodt, 2013; Boschee et al., 2013; O’Connor et al., 2013). A coreference component can improve extraction coverage in cases such as "Russian troops were sighted ... and they attacked ..."

We use the coreference system examined in $\S 5$ for this analysis. To propagate coreference uncertainty, we re-run event extraction on multiple coreference samples generated from the algorithm described in $\S 5.2$, inducing a posterior distribution over the event counts. To isolate the effects of coreference, we use a very simple syntactic dependency system to identify affiliations and events. Assume the availability of dependency parses for a document $d$, a coreference resolution $\mathbf{e}$, and a lexicon of country names, which contains a small set of words $w(c)$ for each country $c$; for example, $w($ FRA $)=\{$ france, french $\}$. The binary function 
$f\left(c, e ; x_{d}\right)$ assesses whether an entity $e$ is affiliated with country $c$ and is described as the agent of an attack, based on document text and parses $x_{d} ; f$ returns true iff both: ${ }^{10}$

- There exists a mention $i \in e$ described as country $c$ : either its head word is in $w(c)$ (e.g. "Americans"), or its head word has an nmod or amod modifier in $w(c)$ (e.g. "American forces", "president of the U.S."); and there is only one unique country $c$ among the mentions in the entity.

- There exists a mention $j \in e$ which is the nsubj or agent argument to the verb "attack" (e.g. "they attacked", "the forces attacked", "attacked by them").

For a given $c$, we first calculate a binary variable for whether there is at least one entity fulfilling $f$ in a particular document,

$$
a\left(d, c, \mathbf{e}_{d}\right)=\bigvee_{e \in \mathbf{e}_{d}} f\left(c, e ; x_{d}\right)
$$

and second, the number of such documents in $d(t)$, the set of New York Times articles published in a given time period $t$,

$$
n\left(t, c, \mathbf{e}_{d(t)}\right)=\sum_{d \in d(t)} a\left(d, c, \mathbf{e}_{d}\right)
$$

These quantities are both random variables, since they depend on e; thus we are interested in the posterior distribution of $n$, marginalizing out $\mathbf{e}$,

$$
P\left(n\left(t, c, \mathbf{e}_{d(t)}\right) \mid x_{d(t)}\right)
$$

If our coreference model was highly certain (only one structure, or a small number of similar structures, had most of the probability mass in the space of all possible structures), each document would have an $a$ posterior near either 0 or 1 , and their sum in Eq. 5 would have a narrow distribution. But if the model is uncertain, the distribution will be wider. Because of the transitive closure, the probability of $a$ is potentially more complex than the single antecedent linking probability between two mentions - the affiliation and attack information can propagate through a long coreference chain.

\subsection{Results}

We tag and parse a 193,403 article subset of the Annotated New York Times LDC corpus (Sandhaus, 2008), which includes articles about world

\footnotetext{
${ }^{10}$ Syntactic relations are Universal Dependencies (de Marneffe et al., 2014); more details for the extraction rules are in the appendix.
}

news from the years 1987 to 2007 (details in appendix). For each article, we run the coreference system to predict 100 samples, and evaluate $f$ on every entity in every sample. ${ }^{11}$ The quantity of interest is the number of articles mentioning attacks in a 3-month period (quarter), for a given country. Figure 6 illustrates the mean and 95\% posterior credible intervals for each quarter. The posterior mean $m$ is calculated as the mean of the samples, and the interval is the normal approximation $m \pm 1.96 \mathrm{~s}$, where $s$ is the standard deviation among samples for that country and time period.

Uncertainty information helps us understand whether a difference between data points is real. In the plots of Figure 6, if we had used a 1-best coreference resolution, only a single line would be shown, with no assessment of uncertainty. This is problematic in cases when the model genuinely does not know the correct answer. For example, the 1993-1996 period of the USA plot (Figure 6, top) shows the posterior mean fluctuating from 1 to 5 documents; but when credible intervals are taken into consideration, we see that model does not know whether the differences are real, or were caused by coreference noise.

A similar case is highlighted at the bottom plot of Figure 6. Here we compare the event counts for Yugoslavia and NATO, which were engaged in a conflict in 1999. Did the New York Times devote more attention to the attacks by one particular side? To a 1-best system, the answer would be yes. But the posterior intervals for the two countries' event counts in mid-1999 heavily overlap, indicating that the coreference system introduces too much uncertainty to obtain a conclusive answer for this question. Note that calibration of the coreference model is important for the credible intervals to be useful; for example, if the model was badly calibrated by being overconfident (too much probability over a small set of similar structures), these intervals would be too narrow, leading to incorrect interpretations of the event dynamics.

Visualizing this uncertainty gives richer information for a potential user of an NLP-based system, compared to simply drawing a line based on a single 1-best prediction. It preserves the genuine uncertainty due to ambiguities the system was unable to resolve. This highlights an alternative use of Finkel et al. (2006)'s approach of sampling multiple NLP pipeline components, which in that work was used to perform joint inference. Instead

\footnotetext{
${ }^{11}$ We obtained similar results using only 10 samples. We also obtained similar results with a different query function, the total number of entities, across documents, that fulfill $f$.
} 
of focusing on improving an NLP pipeline, we can pass uncertainty on to exploratory purposes, and try to highlight to a user where the NLP system may be wrong, or where it can only imprecisely specify a quantity of interest.

Finally, calibration can help error analysis. For a calibrated model, the more uncertain a prediction is, the more likely it is to be erroneous. While coreference errors comprise only one part of event extraction errors (alongside issues in parse quality, factivity, semantic roles, etc.), we can look at highly uncertain event predictions to understand the nature of coreference errors relative to our task. We manually analyzed documents with a $50 \%$ probability to contain an "attack"ing countryaffiliated entity, and found difficult coreference cases.

In one article from late 1990, an "attack" event for IRQ is extracted from the sentence "But some political leaders said that they feared that Mr. Hussein might attack Saudi Arabia". The mention "Mr. Hussein" is classified as IRQ only when it is coreferent with a previous mention "President Saddam Hussein of Iraq"; this occurs only 50\% of the time, since in some posterior samples the coreference system split apart these two "Hussein" mentions. This particular document is additionally difficult, since it includes the names of more than 10 countries (e.g. United States, Saudi Arabia, Egypt), and some of the Hussein mentions are even clustered with presidents of other countries (such as "President Bush"), presumably because they share the "president" title. These types of errors are a major issue for a political analysis task; further analysis could assess their prevalence and how to address them in future work.

\section{Conclusion}

In this work, we argue that the calibration of posterior predictions is a desirable property of probabilistic NLP models, and that it can be directly evaluated. We also demonstrate a use case of having calibrated uncertainty: its propagation into downstream exploratory analysis.

Our posterior simulation approach for exploratory and error analysis relates to posterior predictive checking (Gelman et al., 2013), which analyzes a posterior to test model assumptions; Mimno and Blei (2011) apply it to a topic model.

One avenue of future work is to investigate more effective nonparametric regression methods to better estimate and visualize calibration error, such as Gaussian processes or bootstrapped kernel
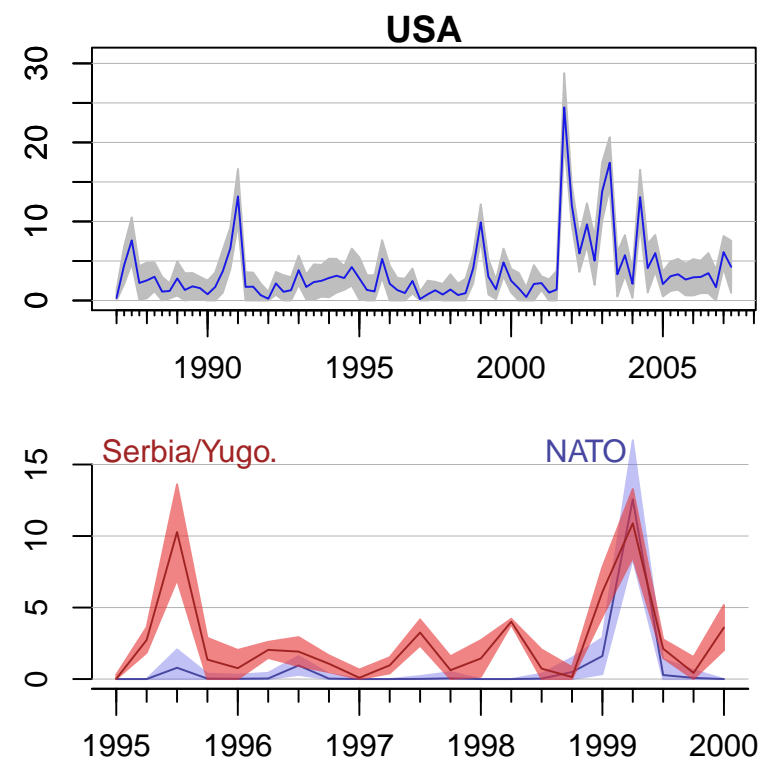

Figure 6: Number of documents with an "attack"ing country per 3-month period, and coreference posterior uncertainty for that quantity. The dark line is the posterior mean, and the shaded region is the $95 \%$ posterior credible interval. More examples in appendix.

density estimation.

Another important question is: what types of inferences are facilitated by correct calibration? Intuitively, we think that overconfidence will lead to overly narrow confidence intervals; but in what sense are confidence intervals "good" when calibration is perfect? Also, does calibration help joint inference in NLP pipelines? It may also assist calculations that rely on expectations, such as inference methods like minimum Bayes risk decoding, or learning methods like EM, since calibrated predictions imply that calculated expectations are statistically unbiased (though the implications of this fact may be subtle). Finally, it may be interesting to pursue recalibration methods, which readjust a non-calibrated model's predictions to be calibrated; recalibration methods have been developed for binary (Platt, 1999; Niculescu-Mizil and Caruana, 2005) and multiclass (Zadrozny and Elkan, 2002) classification settings, but we are unaware of methods appropriate for the highly structured outputs typical in linguistic analysis. Another approach might be to directly constrain CalibErr $=0$ during training, or try to reduce it as a training-time risk minimization or cost objective (Smith and Eisner, 2006; Gimpel and Smith, 2010; Stoyanov et al., 2011; Brümmer and Doddington, 2013).

Calibration is an interesting and important property of NLP models. Further work is necessary to address these and many other questions. 


\section{References}

David Bamman, Brendan O'Connor, and Noah A. Smith. Learning latent personas of film characters. In Proceedings of ACL, 2013.

Paul N. Bennett. Assessing the calibration of naive Bayes' posterior estimates. Technical report, Carnegie Mellon University, 2000.

David M. Blei and Peter I. Frazier. Distance dependent Chinese restaurant processes. The Journal of Machine Learning Research, 12: 2461-2488, 2011.

Elizabeth Boschee, Premkumar Natarajan, and Ralph Weischedel. Automatic extraction of events from open source text for predictive forecasting. Handbook of Computational Approaches to Counterterrorism, page 51, 2013.

Glenn W. Brier. Verification of forecasts expressed in terms of probability. Monthly weather review, 78(1):1-3, 1950.

Jochen Bröcker. Reliability, sufficiency, and the decomposition of proper scores. Quarterly Journal of the Royal Meteorological Society, 135(643):1512-1519, 2009.

Niko Brümmer and George Doddington. Likelihood-ratio calibration using priorweighted proper scoring rules. arXiv preprint arXiv:1307.7981, 2013. Interspeech 2013.

Marie-Catherine de Marneffe, Timothy Dozat, Natalia Silveira, Katri Haverinen, Filip Ginter, Joakim Nivre, and Christopher D. Manning. Universal Stanford dependencies: A cross-linguistic typology. In Proceedings of LREC, 2014.

Morris H. DeGroot and Stephen E. Fienberg. The comparison and evaluation of forecasters. The statistician, pages 12-22, 1983.

Pedro Domingos and Michael Pazzani. On the optimality of the simple Bayesian classifier under zero-one loss. Machine learning, 29(2-3):103130, 1997.

Greg Durrett and Dan Klein. Easy victories and uphill battles in coreference resolution. In EMNLP, pages 1971-1982, 2013.

Greg Durrett and Dan Klein. A joint model for entity analysis: Coreference, typing, and linking. Transactions of the Association for Computational Linguistics, 2:477-490, 2014.

Jenny Rose Finkel, Christopher D. Manning, and Andrew Y. Ng. Solving the problem of cascading errors: Approximate Bayesian inference for linguistic annotation pipelines. In Proceedings of the 2006 Conference on Empirical Methods in Natural Language Processing, pages 618626. Association for Computational Linguistics, 2006.

Andrew Gelman, John B. Carlin, Hal S. Stern, David B. Dunson, Aki Vehtari, and Donald B. Rubin. Bayesian data analysis. Chapman and Hall/CRC, 3rd edition, 2013.

Kevin Gimpel and Noah A. Smith. Rich sourceside context for statistical machine translation. In Proceedings of the Third Workshop on Statistical Machine Translation, pages 9-17, 2008.

Kevin Gimpel and Noah A. Smith. Softmaxmargin CRFs: Training log-linear models with cost functions. In Human Language Technologies: The 2010 Annual Conference of the North American Chapter of the Association for Computational Linguistics, pages 733-736. Association for Computational Linguistics, 2010.

Kevin Gimpel, Dhruv Batra, Chris Dyer, and Gregory Shakhnarovich. A systematic exploration of diversity in machine translation. In Proceedings of the 2013 Conference on Empirical Methods in Natural Language Processing, pages 1100-1111, Seattle, Washington, USA, October 2013. Association for Computational Linguistics. URL http://www. aclweb. org/anthology/D13-1111.

Tilmann Gneiting and Adrian E. Raftery. Strictly proper scoring rules, prediction, and estimation. Journal of the American Statistical Association, 102(477):359-378, 2007.

Joshua Goodman. Parsing algorithms and metrics. In Proceedings of the 34th Annual Meeting of the Association for Computational Linguistics, pages 177-183, Santa Cruz, California, USA, June 1996. Association for Computational Linguistics. doi: 10.3115/981863. 981887. URL http: / /www . aclweb. org/ anthology/P 96-1024.

Aria Haghighi and Dan Klein. Unsupervised coreference resolution in a nonparametric Bayesian model. In Annual Meeting, Association for Computational Linguistics, volume 45, page 848, 2007.

Robert E. Kass, Bradley P. Carlin, Andrew Gelman, and Radford M. Neal. Markov chain Monte Carlo in practice: a roundtable discussion. The American Statistician, 52(2):93-100, 1998. 
Shankar Kumar and William Byrne. Minimum Bayes-risk decoding for statistical machine translation. In Daniel Marcu Susan Dumais and Salim Roukos, editors, HLT-NAACL 2004: Main Proceedings, pages 169-176, Boston, Massachusetts, USA, May 2 - May 7 2004. Association for Computational Linguistics.

Kalev Leetaru and Philip A. Schrodt. GDELT: Global data on events, location, and tone, 19792012. In ISA Annual Convention, volume 2, page 4, 2013.

Jimmy Lin and Alek Kolcz. Large-scale machine learning at Twitter. In Proceedings of the 2012 ACM SIGMOD International Conference on Management of Data, pages 793-804. ACM, 2012.

Michael C. McCord, J. William Murdock, and Branimir K. Boguraev. Deep parsing in Watson. IBM Journal of Research and Development, 56 (3.4):3-1, 2012.

David Mimno and David Blei. Bayesian checking for topic models. In Proceedings of the 2011 Conference on Empirical Methods in Natural Language Processing, pages 227-237, Edinburgh, Scotland, UK., July 2011. Association for Computational Linguistics. URL http://www.aclweb.org/ anthology/D11-1021.

Makoto Miwa, Sampo Pyysalo, Tadayoshi Hara, and Jun'ichi Tsujii. Evaluating dependency representations for event extraction. In Proceedings of the 23rd International Conference on Computational Linguistics (Coling 2010), pages 779-787, Beijing, China, August 2010. Coling 2010 Organizing Committee. URL http://www.aclweb.org/ anthology/C10-1088.

Allan H. Murphy and Robert L. Winkler. A general framework for forecast verification. Monthly Weather Review, 115(7):1330-1338, 1987.

Andrew Ng and Michael Jordan. On discriminative vs. generative classifiers: A comparison of logistic regression and naive Bayes. Advances in neural information processing systems, 14: 841, 2002.

Alexandru Niculescu-Mizil and Rich Caruana. Predicting good probabilities with supervised learning. In Proceedings of the 22nd International Conference on Machine Learning, pages 625-632, 2005.
Brendan O'Connor, Brandon Stewart, and Noah A. Smith. Learning to extract international relations from political context. In Proceedings of ACL, 2013.

Naoaki Okazaki. Crfsuite: a fast implementation of conditional random fields (CRFs), 2007. URL http://www. chokkan.org/ software/crfsuite/.

F. Pedregosa, G. Varoquaux, A. Gramfort, V. Michel, B. Thirion, O. Grisel, M. Blondel, P. Prettenhofer, R. Weiss, V. Dubourg, J. Vanderplas, A. Passos, D. Cournapeau, M. Brucher, M. Perrot, and E. Duchesnay. Scikit-learn: Machine learning in Python. Journal of Machine Learning Research, 12:2825-2830, 2011.

John Platt. Probabilistic outputs for support vector machines and comparisons to regularized likelihood methods. In Advances in large margin classifiers. MIT Press (2000), $1999 . \quad$ URL http: //research.microsoft.com/ pubs / 69187/svmprob.ps.gz.

Sameer Pradhan, Lance Ramshaw, Mitchell Marcus, Martha Palmer, Ralph Weischedel, and Nianwen Xue. CoNLL-2011 shared task: Modeling unrestricted coreference in Ontonotes. In Proceedings of the Fifteenth Conference on Computational Natural Language Learning: Shared Task, pages 1-27. Association for Computational Linguistics, 2011.

Jonathon Read. Using emoticons to reduce dependency in machine learning techniques for sentiment classification. In Proceedings of the ACL Student Research Workshop, pages 43-48. Association for Computational Linguistics, 2005.

Evan Sandhaus. The New York Times Annotated Corpus. Linguistic Data Consortium, LDC2008T19, 2008.

Philip A. Schrodt. Precedents, progress, and prospects in political event data. International Interactions, 38(4):546-569, 2012.

Philip A. Schrodt, Shannon G. Davis, and Judith L. Weddle. KEDS - a program for the machine coding of event data. Social Science Computer Review, 12(4):561 -587, December 1994. doi: 10.1177/089443939401200408. URL http://ssc.sagepub.com/ content/12/4/561.abstract.

Sameer Singh, Sebastian Riedel, Brian Martin, Jiaping Zheng, and Andrew McCallum. Joint inference of entities, relations, and coreference. 
In Proceedings of the 2013 Workshop on Automated Knowledge Base Construction, pages 16. ACM, 2013.

David A. Smith and Jason Eisner. Minimum risk annealing for training log-linear models. In Proceedings of the COLING/ACL 2006 Main Conference Poster Sessions, pages 787-794, Sydney, Australia, July 2006. Association for Computational Linguistics. URL http: //www . aclweb.org/anthology/p 06-2101.

Veselin Stoyanov, Alexander Ropson, and Jason Eisner. Empirical risk minimization of graphical model parameters given approximate inference, decoding, and model structure. In International Conference on Artificial Intelligence and Statistics, pages 725-733, 2011.

Kristina Toutanova, Aria Haghighi, and Christopher D. Manning. A global joint model for semantic role labeling. Computational Linguistics, 34(2):161-191, 2008.

John W. Tukey. Curves as parameters, and touch estimation. In Proceedings of the Fourth Berkeley Symposium on Mathematical Statistics and Probability, Volume 1: Contributions to the Theory of Statistics, pages 681-694, Berkeley, Calif., 1961. University of California Press. URL http://projecteuclid. org/euclid.bsmsp/1200512189.

Ashish Venugopal, Andreas Zollmann, Noah A. Smith, and Stephan Vogel. Wider pipelines: Nbest alignments and parses in MT training. In Proceedings of AMTA, 2008.

Larry Wasserman. All of nonparametric statistics. Springer Science \& Business Media, 2006.

Bianca Zadrozny and Charles Elkan. Transforming classifier scores into accurate multiclass probability estimates. In Proceedings of KDD, pages 694-699. ACM, 2002. 